\title{
Systems of differential equations with implicit impulses and fully nonlinear boundary conditions
}

\author{
Yawei Song and Bevan Thompson*
}

\section{"Correspondence:}

hbt@maths.edu.au

School of Mathematics and Physics,

The University of Queensland,

Brisbane, Queensland 4072,

Australia

\begin{abstract}
We show that systems of second-order ordinary differential equations, $x^{\prime \prime}=f\left(t, x, x^{\prime}\right)$, subject to compatible nonlinear boundary conditions and impulses, have a solution $x$ such that $(t, x(t))$ lies in an admissible bounding subset of $[0,1] \times \mathbb{R}^{n}$ when $f$ satisfies a Hartman-Nagumo growth bound with respect to $x^{\prime}$. We reformulate the problem as a system of nonlinear equations and apply Leray-Schauder degree theory. We compute the degree by homotopying to a new system of nonlinear equations based on the simpler system of ordinary differential equations, $x^{\prime \prime}=M_{0} L(x-v)$, subject to Picard boundary conditions and impulses and using the Leray index theorem. Our proof is simpler than earlier existence proofs involving nonlinear boundary conditions without impulses and requires weak assumptions on $f$.
\end{abstract}

MSC: 34A37; 34A34; 34B15

Keywords: boundary value problems; nonlinear boundary conditions; impulses; Leray-Schauder degree

\section{Introduction}

Let $q \in \mathbb{N}$, the natural numbers,

$$
Q=\left\{t_{1}, \ldots, t_{q}: 0=t_{0}<t_{1}<\cdots<t_{q}<t_{q+1}=1\right\} .
$$

$J_{0}=\left[t_{0}, t_{1}\right]$ and $J_{k}=\left(t_{k}, t_{k+1}\right]$ for $1 \leq k \leq q$. We call $Q$ a division of the interval $[0,1]$.

We consider the system of second-order ordinary differential equations

$$
x^{\prime \prime}=f\left(t, x, x^{\prime}\right), \quad t \in[0,1] \backslash Q
$$

subject to very general nonlinear boundary conditions of the form

$$
g_{0}\left(x(0), x(1), x^{\prime}(0), x^{\prime}(1)\right)=(0,0)
$$

and very general nonlinear implicit impulses of the form

$$
g_{k}\left(x\left(t_{k}^{+}\right), x\left(t_{k}^{-}\right), x^{\prime}\left(t_{k}^{+}\right), x^{\prime}\left(t_{k}^{-}\right)\right)=(0,0), \quad k=1, \ldots, q,
$$

@2013 Song and Thompson; licensee Springer. This is an Open Access article distributed under the terms of the Creative Commons Attribution License (http://creativecommons.org/licenses/by/2.0), which permits unrestricted use, distribution, and reproduction in any medium, provided the original work is properly cited. 
where

$$
f:[0,1] \times \mathbb{R}^{2 n} \rightarrow \mathbb{R}^{n}
$$

satisfies $\left.f\right|_{J_{k} \times \mathbb{R}^{2 n}}$ has an extension to $f_{k} \in C\left(\bar{J}_{k} \times \mathbb{R}^{2 n} ; \mathbb{R}^{n}\right)$ and

$$
g_{k}=\left(g_{k, 1}, g_{k, 2}\right) \in C\left(\mathbb{R}^{2 n} \times \mathbb{R}^{2 n} ; \mathbb{R}^{2 n}\right)
$$

for $0 \leq k \leq q$. Our fully nonlinear boundary conditions (2) include the Picard, periodic, and Neumann boundary conditions as special cases. We establish a general existence result for solutions lying in an admissible bounding set for the system of ordinary differential equations (1) satisfying boundary conditions (2) and impulses (3).

Our result is closely related to those of Thompson [1] and of Kongson et al. [2]. In [1] and [2], the authors established existence results for systems of second-order ordinary differential equations in more general bounding sets and subject to general boundary conditions (2) but not subject to impulses. Moreover, the proof in [1] is incomplete as it fails to establish the required derivative bounds; these appear to require more assumptions on the Hartman-Nagumo growth bound than we assume here. Although our bounding sets are more restrictive than those in [2], our proof is much simpler than theirs. In particular, the ideas introduced in our proof offer a fresh starting point for further work aimed at identifying the natural and most general concept of a bounding set and with this the natural and most general existence results possible for system (1) subject to nonlinear boundary conditions (2).

Earlier works on boundary value problems homotopies the original problem (1), plus nonlinear boundary conditions (2), to $x^{\prime \prime}(t)=0$ plus the Picard boundary conditions; see, for example, [3]. This requires $f$ to be redefined for $(t, x)$ outside the admissible bounding set in such a way that solutions to the associated boundary value problem lie in the admissible bounding set. This in turn imposes restrictive assumptions on $f$ and the associated bounding set. A key to our new idea is the observation that it suffices to homotopy our associated system of nonlinear equations to a new system of nonlinear equations associated with the simpler system $x^{\prime \prime}(t)=M_{0} L[x-v(t)]$ subject to Picard boundary conditions and impulses. This is uniquely solvable with the solution lying in the admissible bounding set. We use the Leray index theorem and the multiplication theorem to show that the degree of the associated nonlinear equation is not zero. Using our homotopies, we do not need to redefine the system outside the admissible bounding set. In the current work, we require the bounding set to be $\left\{(t, x) \in[0,1] \times \mathbb{R}^{n}: r(t, x)<0\right\}$, where $r:[0,1] \times \mathbb{R}^{n} \rightarrow \mathbb{R}$ and $r(t, \cdot)$ is strongly convex as a function of $x$ (see Remark 1(i)).

A further motivation for our work comes from the paper by Cabada and Thompson [4] for a single equation with impulses. Recently, many papers devoted to the study of boundary value problems for nonlinear differential equations with impulses have appeared because of their wide applicability and associated rich theory. In the literature one can find different kinds of existence results for first-order [5, 6], second-order [7-9], and higherorder $[10,11]$ ordinary differential equations with periodic boundary conditions and impulses. In addition, some existence results for first-order impulsive differential equation with nonlinear boundary conditions can be found in [12-15]. In the papers [4, 16, 17], the $\phi$-Laplacian and $\varphi$-Laplacian equations with impulses are considered. 
This paper is organized as follows. In Section 2, we introduce the notation and definitions that we use in this paper. We give the definition of compatible boundary conditions and introduce our definition of compatible impulses in Section 3. In Section 4, we present the Nagumo-type condition that we use in our existence result to $a$ priori bound the derivative of solutions. Section 5 is principally devoted to our main result where we prove that there are solutions to (1), (2), and (3) lying in an admissible bounding set. In Section 6, we present an example.

\section{Notation and definitions}

In this section, we present the notation, definitions, and assumptions that we use to obtain a priori bounds on solutions.

Let $H$ denote finite or infinite dimensional Hilbert spaces. For a bounded subset $V$ of $H$, let $V^{\circ}$ denote its interior, $\partial V$ its boundary and $\bar{V}$ its closure. For a bounded subset $U$ of $[0,1] \times \mathbb{R}^{n}$ and $t \in[0,1]$, let $U(t)$ denote its $t$-cross section and $\partial U(t)$ denote the boundary of $U(t)$ in $\mathbb{R}^{n}$. Thus $U(t)=\left\{x \in \mathbb{R}^{n}:(t, x) \in U\right\}$. Let $\partial_{C} U$ denote the curved boundary of $U$, so $\partial_{C} U=\bigcup_{t \in[0,1]} \partial U(t)$ excludes the sets $\{0\} \times U^{\circ}(0)$ and $\{1\} \times U^{\circ}(1)$ from $\partial U$. For $x \in \mathbb{R}$, $|x|$ denotes the absolute value of $x$. For $x=\left(x_{1}, \ldots, x_{n}\right) \in \mathbb{R}^{n}$ and $y=\left(y_{1}, \ldots, y_{n}\right) \in \mathbb{R}^{n}, x^{T}$ denotes the transpose of $x$ while $x \cdot y$ denotes the scalar product of $x$ and $y$. Let $\mathcal{I}$ denote the identity on $H$ so $\mathcal{I}(x)=x$ for all $x$. If $X$ is a Banach space and $A \subset H$, then $C^{m}(A ; X)$ denotes the space of $m$-times continuously differentiable functions from $A$ to $X$ with a finite norm. In the case of continuous functions, we omit the $m$, while in the case of realvalued functions, we omit the $X$.

Let $J \subset \mathbb{R}$ be an interval. For $r \in C^{2}\left(J \times \mathbb{R}^{n}\right)$, let $r_{t}(t, x)$ denote the partial derivative with respect to $t, r_{x}(t, x)$ denote the gradient, and $r_{x x}(t, x)$ denote the matrix of second-order partial derivatives of $r$ with respect to $x$.

The norm on $C^{m}\left(J ; \mathbb{R}^{n}\right)$ is given by

$$
\|u\|_{c^{m}(J)}=\sup _{k \leq m ; t \in J}\left\|u^{(k)}(t)\right\|
$$

where $u^{(k)}$ denotes the $k$ th derivative of $u$. By abuse of notation, we abbreviated $C^{m}\left(J ; \mathbb{R}^{n}\right)$ to $C^{m}(J)$. Further we will abbreviate $\|u\|_{C^{m}(J)}$ to $\|u\|$ when the meaning is clear from the context.

For $\tau \in[0,1)$, let $u^{(l)}\left(\tau^{+}\right)=\lim _{t \rightarrow \tau^{+}} u^{(l)}(t)$ and for $\tau \in(0,1]$, let $u^{(l)}\left(\tau^{-}\right)=\lim _{t \rightarrow \tau-} u^{(l)}(t)$ for $0 \leq l \leq m$. To simplify statements of results, set $u^{(l)}\left(1^{+}\right)=u^{(l)}(1)$ and $u^{(l)}\left(0^{-}\right)=u^{(l)}(0)$ for $0 \leq l \leq m$, where $u^{(l)}(1)$ and $u^{(l)}(0)$ are the appropriate one-sided derivatives.

In order to define the concept of solution for our problem, we consider the following sets. Let

$$
\begin{aligned}
C_{Q}^{m}= & \left\{u:[0,1] \rightarrow \mathbb{R}^{n}:\left.u\right|_{J_{k}} \in C^{m}\left(J_{k}\right), u^{(m)}\left(t_{k}^{+}\right)\right. \\
& \text {exist for } k=1, \ldots, q\} .
\end{aligned}
$$

All our limits are assumed to be $\mathbb{R}^{n}$-valued when they exist. Thus, for $u \in C_{Q}^{m}, u^{(l)}\left(t_{k}^{ \pm}\right)$ exists for $k=0, \ldots, q+1, l=0, \ldots, m$. Note that $C_{Q}^{0}$ is defined in the obvious way. Thus we may identify $x \in C_{Q}^{m}$ with $\tilde{x}=\left(x_{0}, \ldots, x_{q}\right) \in \prod_{k=0}^{q} C^{m}\left(\bar{J}_{k}\right)$, where $\tilde{x}(t)=x_{k}(t)$, for all $t \in J_{k}$. 
By abuse of notation, we will denote $\tilde{x}$ by $x$ where the meaning is clear from the context. Further we define a norm on $C_{Q}^{m}$ by

$$
\|x\|_{C_{Q}^{m}}=\max _{k}\|x\|_{C^{m}\left(J_{k}\right)}
$$

If $A$ is a bounded open subset of $H, \mathcal{G}(x)=x+\mathcal{K}(x)$, where $\mathcal{K} \in C(\bar{A}, H), \mathcal{K}(\bar{A})$ has compact closure and $p \in H \backslash \mathcal{G}(\partial A)$, then $d(\mathcal{G}, A, p)$ denotes the Leray-Schauder degree of $\mathcal{G}$ on $A$ at $p$. In the special case that $H=\mathbb{R}^{n}$ and $\mathcal{G} \in C\left(\bar{A}, \mathbb{R}^{n}\right), p \in \mathbb{R}^{n} \backslash \mathcal{G}(\partial A), d(\mathcal{G}, A, p)$ is the Brouwer degree.

By a solution $x$ we mean a function $x \in C_{Q}^{2}$ satisfying (1) for all $t \in[0,1] \backslash Q$, (2) and (3). We look for solutions to problem (1) together with the fully nonlinear boundary conditions (2) and impulses (3) in the following admissible bounding set which provides a priori bounds on solutions to (1).

Definition 1 Let $\Omega \subset[0,1] \times \mathbb{R}^{n}$ be a bounded set and $v \in C_{Q}^{2}$. We call $(\Omega, v)$ an admissible bounding set for (1) if it has the following properties:

(i) There is $r:[0,1] \times \mathbb{R}^{n} \rightarrow \mathbb{R}$ such that

(a) $\left.r\right|_{J_{k} \times \mathbb{R}^{n}}$ can be uniquely extended to $r_{k} \in C^{2}\left(\bar{J}_{k} \times \mathbb{R}^{n}\right)$ for all $0 \leq k \leq q$;

(b) $\Omega:=\left\{(t, x) \in[0,1] \times \mathbb{R}^{n}: r(t, x)<0\right\}$;

(c) $\sum_{i, j=1}^{n} r_{x_{i} x_{j}}(t, x) \xi_{i} \xi_{j} \geq \Theta\|\xi\|^{2}$ for some constants $\Theta>0$, all $\xi \in \mathbb{R}^{n}$ and $(t, x) \in \Omega$;

(ii) There is $\varepsilon>0$ such that $B_{\varepsilon}\left(v_{k}(t)\right) \subseteq \Omega_{k}(t)$, where $\Omega_{k}:=\left\{(t, x) \in \bar{J}_{k} \times \mathbb{R}^{n}: r_{k}(t, x)<0\right\}$ for all $0 \leq k \leq q$;

(iii) If $t \in(0,1) \backslash Q, p \in \mathbb{R}^{n}, r(t, u)=0$ and $r^{\prime}(t, u, p)=0$, then

$$
r_{f}^{\prime \prime}(t, u, p)>0,
$$

where

$$
\begin{aligned}
& r^{\prime}(t, u, p)=r_{t}(t, u)+r_{x}^{T}(t, u) p, \\
& r_{f}^{\prime \prime}(t, u, p)=r_{t t}(t, u)+2 r_{t x}^{T}(t, u) p+p^{T} r_{x x}(t, u) p+r_{x}^{T}(t, u) f(t, u, p)
\end{aligned}
$$

(iv) $\left\|r_{x}(t, x)\right\| \geq c>0$ for all $(t, x) \in \partial_{C} \Omega$ and some constant $c>0$.

\section{Remark 1}

(i) A function $r \in C^{2}\left(\mathbb{R}^{n}\right)$ is strongly convex iff for some constants $\Theta>0$,

$$
\sum_{i, j=1}^{n} r_{x x}(x) \xi_{i} \xi_{j} \geq \Theta\|\xi\|^{2}
$$

for $x, \xi \in \mathbb{R}^{n}$ (see Part 4 in [18]). If $r \in C^{2}\left(\mathbb{R}^{n}\right)$ satisfies (6), then $r$ is uniformly convex, see Appendix B.1. in [19]. Moreover, $r \in C^{2}\left(\mathbb{R}^{n}\right)$ satisfies (6) when $\Theta=0$ iff $r$ is convex (see Appendix B.1. in [19]). From the definition of convex function, it is easy to see that

$$
\Omega(t)=\left\{x \in \mathbb{R}^{n}: r(t, x)<0\right\}
$$

is a convex set for $t \in[0,1]$. 
It follows from Definition 1(i)(c) that for $(t, x, p) \in \bar{\Omega} \times \mathbb{R}^{n}$,

$$
\begin{aligned}
r_{0}^{\prime \prime}(t, x, p) & =p^{T} r_{x x}(t, x) p+2 p^{T} r_{t x}(t, x)+r_{t t}(t, x) \\
& \geq \Theta\|p\|^{2}-2 D\|p\|-D \\
& >-K_{1}
\end{aligned}
$$

where $D=\sup _{(t, x) \in \Omega}\left\{\left\|r_{t x}(t, x)\right\|,\left\|r_{t t}(t, x)\right\|\right\}$ and $K_{1}>0$.

(ii) It follows from Definition 1(i)(a), (ii) and (iv) that

$$
\begin{aligned}
& \left\|r_{x}(t, x)\right\| \geq c>0, \\
& (x-v(t)) \cdot r_{x}(t, x)>\eta\left\|r_{x}(t, x)\right\| \quad \text { and hence } \\
& (x-v(t)) \cdot r_{x}(t, x)>\eta c>0
\end{aligned}
$$

for all $(t, x) \in \bigcup_{k} \partial_{C} \Omega_{k}$ and some $\eta>0$.

Set

$$
\Delta_{0}=\Omega(0) \times \Omega(1), \quad \Delta_{k}=\Omega_{k}\left(t_{k}\right) \times \Omega_{k-1}\left(t_{k}\right)=\Omega\left(t_{k}^{+}\right) \times \Omega\left(t_{k}^{-}\right)
$$

for $1 \leq k \leq q$, where $\Omega\left(t_{k}^{+}\right)=\Omega_{k}\left(t_{k}\right)$ and $\Omega\left(t_{k}^{-}\right)=\Omega_{k-1}\left(t_{k}\right)$. Let

$$
R_{1}=\sup \{\|x-v(t)\|:(t, x) \in \Omega\}+1 ; \quad R_{2}=R_{1}+\sup \|v(t)\| .
$$

We assume that $f$ satisfies the following conditions.

Definition 2 Let $(\Omega, v)$ be an admissible bounding set for (1). We say that $f$ satisfies the Hartman-Nagumo condition on $\Omega$ if:

(i) $\left.f\right|_{J_{k} \times \mathbb{R}^{2 n}}$ has an extension to $f_{k} \in C\left(\bar{J}_{k} \times \mathbb{R}^{2 n}\right)$;

(ii) $\|f(t, x, p)\| \leq \Phi(\|p\|)$ for all $(t, x, p) \in \Omega \times \mathbb{R}^{n}$, where

$$
\int^{\infty} \frac{s}{\Phi(s)} d s=\infty
$$

(iii) $\|f(t, x, p)\| \leq M r_{f}^{\prime \prime}(t, x, p)+K$ for all $(t, x, p) \in \Omega \times \mathbb{R}^{n}$, where $M$ and $K$ are nonnegative constants and $r_{f}^{\prime \prime}$ is given by (5).

Remark 2 If conditions (ii) and (iii) above are satisfied, a solution $x$ of (1) with $(t, x(t)) \in \Omega$ satisfies the Hartman-Nagumo inequality (see the second paragraph on p.702 in [20]).

\section{Compatibility}

Following [1], we give the definition of compatible boundary conditions and introduce the definition of compatible impulses. These are simple, degree-based relationships between the boundary conditions, the impulses, and the associated admissible bounding set. For more information on compatibility of boundary conditions, we refer the reader to $[1,21]$, and [4, Definition 14]. 
Definition 3 For $1 \leq k \leq q$, we call the vector field $\Psi_{k}=\left(\psi_{k}^{0}, \psi_{k}^{1}\right) \in C\left(\bar{\Delta}_{k} ; \mathbb{R}^{2 n}\right)$ strongly inwardly pointing on $\Delta_{k}$ if for all $\left(C_{k}, D_{k}\right) \in \bar{\Delta}_{k}$,

$$
\begin{aligned}
& r_{k}^{\prime}\left(t_{k}, C_{k}, \psi_{k}^{0}\left(C_{k}, D_{k}\right)\right)<0 \quad \text { for all } C_{k} \in \partial \Omega_{k}\left(t_{k}\right), \\
& r_{k-1}^{\prime}\left(t_{k}, D_{k}, \psi_{k}^{1}\left(C_{k}, D_{k}\right)\right)>0 \quad \text { for all } D_{k} \in \partial \Omega_{k-1}\left(t_{k}\right),
\end{aligned}
$$

where $\Delta_{k}, \Omega_{k}\left(t_{k}\right), \Omega_{k-1}\left(t_{k}\right)$ are given in (9). We call the vector field $\Psi_{0}=\left(\psi_{0}^{0}, \psi_{0}^{1}\right) \in$ $C\left(\bar{\Delta}_{0} ; \mathbb{R}^{2 n}\right)$ strongly inwardly pointing on $\Delta_{0}:=\Omega_{0}(0) \times \Omega_{q}(1)$ if for all $\left(C_{0}, D_{0}\right) \in \bar{\Delta}_{0}$,

$$
\begin{array}{ll}
r_{0}^{\prime}\left(0, C_{0}, \psi_{0}^{0}\left(C_{0}, D_{0}\right)\right)<0 & \text { for all } C_{0} \in \partial \Omega_{0}(0), \\
r_{q}^{\prime}\left(1, D_{0}, \psi_{0}^{1}\left(C_{0}, D_{0}\right)\right)>0 & \text { for all } D_{0} \in \partial \Omega_{q}(1),
\end{array}
$$

where $r_{k}(k=0, \ldots, q)$ is the extension to $\bar{J}_{k}$ of $\left.r\right|_{J_{k}^{\circ}}$ and $r_{k}^{\prime}$ is given by (4). From (9), $\Omega_{0}(0)=\Omega(0) ; \Omega_{q}(1)=\Omega(1)$. For $k=0, \ldots, q$, we call $\Psi_{k}$ inwardly pointing on $\Delta_{k}$ if the above inequalities are weak.

In what follows, where there is a strongly inwardly pointing vector field $\Psi_{k}$ on $\bar{\Delta}_{k}$ for all $0 \leq k \leq q$, then $\mathcal{G}_{k}$ is defined by

$$
\mathcal{G}_{k}\left(C_{k}, D_{k}\right)=g_{k}\left(\left(C_{k}, D_{k}\right) ; \Psi_{k}\left(C_{k}, D_{k}\right)\right)
$$

for all $\left(C_{k}, D_{k}\right) \in \bar{\Delta}_{k}, 0 \leq k \leq q$.

The following definition is a variant of Definition 2.5 given in [1].

Definition 4 Let $0 \leq k \leq q$ and $g_{k} \in C\left(\bar{\Delta}_{k} \times \mathbb{R}^{2 n} ; \mathbb{R}^{2 n}\right)$. We say $g_{k}$ is strongly compatible with $\Omega$ if

$$
g_{k}\left(\left(C_{k}, D_{k}\right),\left(u_{k}, v_{k}\right)\right) \neq 0
$$

for all $\left(C_{k}, D_{k}, u_{k}, v_{k}\right) \in \bar{\Delta}_{k} \times \mathbb{R}^{2 n}$ such that

$$
C_{k} \in \partial \Omega_{k}\left(t_{k}\right) \quad \text { and } \quad r_{k}^{\prime}\left(t_{k}, C_{k}, u_{k}\right)<0
$$

and/or

$$
D_{k} \in \partial \Omega_{k-1}\left(t_{k}\right) \quad \text { and } \quad r_{k-1}^{\prime}\left(t_{k}, D_{k}, v_{k}\right)>0
$$

and

$$
d\left(\mathcal{G}_{k}, \Delta_{k}, 0\right) \neq 0
$$

for any strongly inwardly pointing vector field $\Psi_{k}$ on $\bar{\Delta}_{k}$.

For $0 \leq k \leq q$, we say that $g_{k}$ is compatible with $\Omega$ if there is a sequence $g_{k_{i}} \in C\left(\bar{\Delta}_{k} \times\right.$ $\left.\mathbb{R}^{2 n} ; \mathbb{R}^{2 n}\right)$ strongly compatible with $\Omega$ and converging uniformly to $g_{k}$ on compact subsets of $\bar{\Delta}_{k} \times \mathbb{R}^{2 n}$. 


\section{Nagumo-type conditions}

In the literature, there are many variants of the 'Nagumo condition' which are used to establish a priori bounds on the derivative of bounded solutions.

We use the following variant of Lemma 4.1 in [2].

Lemma 1 Let $\Phi \in C([0, \infty) ;[0, \infty))$ satisfy

$$
\int^{\infty} \frac{s}{\Phi(s)} d s=\infty
$$

and $r$ be given in Definition 1(i). Let $x$ be a solution of $(1)$ satisfying $r(t, x(t)) \leq 0$. Assume that

$$
\begin{aligned}
& \|f(t, x, p)\| \leq M_{1} \Phi(\|p\|), \\
& \|f(t, x, p)\| \leq M_{2} r_{f}^{\prime \prime}(t, x, p)+K
\end{aligned}
$$

for $(t, x)$ such that $r(t, x) \leq 0$ and $p \in \mathbb{R}^{n}$, where $M_{1}, M_{2}$, and $K$ are nonnegative constants and $r_{f}^{\prime \prime}$ is given by (5). Then there exists $N=N\left(r, M_{1}, M_{2}, K, \Phi\right)>0$ such that $\left\|x^{\prime}(t)\right\|<N$.

Proof Since $r$ is given in Definition 1(i), then $\|x\| \leq R_{2}$ when $r(t, x) \leq 0$, where $R_{2}$ is given in (10). Thus the proof of Lemma 5.2 of Hartman [22] carries over to our case on $\bar{\Omega}_{k}$, and it follows that $\left\|x^{\prime}(t)\right\|<N_{k}\left(r, M_{1}, M_{2}, K, \Phi\right)$ for $t \in \bar{J}_{k}$. Thus $\left\|x^{\prime}(t)\right\|<N$ for $t \in[0,1]$, where $N=\max _{0 \leq k \leq q} N_{k}\left(r, M_{1}, M_{2}, K, \Phi\right)$.

Remark 3 The function $\Phi \equiv 1$ satisfies (13).

\section{The main result}

In this section, we present the main result of this paper. We prove the existence of at least one solution to nonlinear problem (1), (2), and (3) lying in an admissible bounding set. To achieve this, we turn our impulsive boundary value problem into an equivalent nonlinear equation and use Leray-Schauder degree theory. We compute the degree using three homotopies, the Leray index theorem and the multiplication theorem.

The first homotopy involves $\mathcal{S}(x, C, D, \lambda)=\left(\mathcal{S}_{0}, \ldots, \mathcal{S}_{q}\right)$, where

$$
\begin{aligned}
\mathcal{S}_{k}= & g_{k}\left(\left(C_{k}, D_{k}\right) ; \lambda\left(x^{\prime}(0), x^{\prime}(1)\right)\right. \\
& \left.+(1-\lambda) \Psi_{k}\left(C_{k}, D_{k}\right)\right) .
\end{aligned}
$$

The second and third homotopies are constructed using one-parameter families of systems of ordinary differential equations.

We construct our first family of systems of differential equations using $f_{0}$ defined below. Let $\Phi, K_{1}, M$, and $K$ be given in Lemma 1 , Remark 1 , and Definition 2, respectively. Let

$$
f_{0}(t, x, p)=M_{0} \min \{L, \Phi(\|p\|)\}[x-v(t)]
$$


for $(t, x, p) \in[0,1] \times \mathbb{R}^{2 n}$, where

$$
\begin{aligned}
M_{0}:= & \inf \left\{a \geq 1: a[x-v(t)] \cdot r_{x}(t, x) \geq\left\|r_{x}(t, x)\right\|,(t, x) \in \partial_{C} \Omega\right\}, \\
L:= & \inf \left\{b: M_{0} b[x-v(t)] \cdot r_{x}(t, x)>K_{1} \text { for }(t, x) \in \partial_{C} \Omega\right. \text { and } \\
& \left.M_{0} b \varepsilon>\left\|v^{\prime \prime}(t)\right\| \text { for all } t \in[0,1]\right\},
\end{aligned}
$$

and $\varepsilon$ is given below. Firstly, we consider $M_{2}>M>0$ where $M$ is given in Definition 2. For case $M=0$, see Remark 5. Let

$$
\begin{aligned}
K_{2}:= & \inf \left\{d>\frac{K M_{2}}{M}: M_{0} L\|x-v(t)\| \leq M_{2} r_{0}^{\prime \prime}(t, x, p)+d\right. \\
& \left.+M_{2} M_{0} \min \{L, \Phi(\|p\|)\} r_{x}(t, x) \cdot[x-v(t)], \forall(t, x, p) \in \bar{\Omega} \times \mathbb{R}^{n}\right\} .
\end{aligned}
$$

\section{Remark 4}

(i) It follows that $\left.f_{0}\right|_{J_{k} \times \mathbb{R}^{2 n}}$ has a continuous extension to $\bar{J}_{k} \times \mathbb{R}^{2 n}$.

(ii) It follows from Remark 1 that $M_{0}, K_{2}$, and $L$ are well defined when $M>0$ where $M$ is given in Definition 2 .

For $\lambda \in[0,1]$, we define $f_{\lambda}:[0,1] \times \mathbb{R}^{2 n} \rightarrow \mathbb{R}^{n}$ by

$$
f_{\lambda}(t, x, p)=\lambda f(t, x, p)+(1-\lambda) f_{0}(t, x, p)
$$

where $f_{0}$ and $f$ are given in (14) and (1), respectively.

We consider the system

$$
x^{\prime \prime}=f_{\lambda}\left(t, x, x^{\prime}\right) \quad \text { for all } t \in[0,1] \backslash Q \text {. }
$$

Lemma 2 Let $(\Omega, v)$ be an admissible bounding set for (1) and assume that $f$ satisfies the Hartman-Nagumo condition and that $f_{\lambda}$ is given by (18). Then for $(t, x, p) \in \bar{\Omega} \times \mathbb{R}^{n}$,

$$
\begin{aligned}
\left\|f_{\lambda}(t, x, p)\right\| & \leq M_{1} \Phi(\|p\|), \\
\left\|f_{\lambda}(t, x, p)\right\| & \leq M_{2} r_{f_{\lambda}}^{\prime \prime}(t, x, p)+K_{2},
\end{aligned}
$$

where $\Phi$ is given in Lemma $1, K_{2}$ is given in (17) and $r_{f}^{\prime \prime}$ is given by (5) and $M_{1}, M_{2}$ are nonnegative numbers.

If $x$ is a solution of (19) with $(t, x) \in \bar{\Omega}$, then $\left\|x^{\prime}(t)\right\|<N$ where $N$ is given in Lemma 1 .

Moreover, if $t \in(0,1) \backslash Q, p \in \mathbb{R}^{n}, r(t, x)=0$, and $r^{\prime}(t, x, p)=0$, then $r_{f_{\lambda}}^{\prime \prime}(t, x, p)>0$.

Proof It follows from (17) that

$$
\begin{aligned}
\left\|f_{0}(t, x, p)\right\| & \leq M_{0} L\|x-v(t)\| \\
& \leq M_{2}\left\{r_{0}^{\prime \prime}+r_{x} \cdot M_{0} \min \{L, \Phi(\|p\|)\}(x-v(t))\right\}+K_{2} \\
& =M_{2} r_{f_{0}}^{\prime \prime}+K_{2}
\end{aligned}
$$


for $(t, x, p) \in \bar{\Omega} \times \mathbb{R}^{n}$. Since $f$ satisfies the Hartman-Nagumo condition, thus for $(t, x, p) \in$ $\bar{\Omega} \times \mathbb{R}^{n}$, and $R_{1}$ is given in (10), it is easy to see that

$$
\left\|f_{\lambda}\right\|=\left\|\lambda f(t, x, p)+(1-\lambda) f_{0}(t, x, p)\right\| \leq M_{0} R_{1} \Phi(\|p\|):=M_{1} \Phi(\|p\|) .
$$

Since $M_{2}>M>0$, it follows from (17) that

$$
\begin{aligned}
\left\|f_{\lambda}\right\| & =\left\|\lambda f(t, x, p)+(1-\lambda) f_{0}(t, x, p)\right\| \\
& \leq \lambda\left[M r_{f}^{\prime \prime}+K\right]+(1-\lambda)\left[M_{2} r_{f_{0}}^{\prime \prime}+K_{2}\right] \\
& =M_{2} r_{f_{\lambda}}^{\prime \prime}+K_{2} .
\end{aligned}
$$

If $x$ is a solution of (19) with $(t, x) \in \bar{\Omega}$, it follows from Lemma 1 that $\left\|x^{\prime}(t)\right\|<N$ where $N$ is given in Lemma 1.

From Definition 1(iii), if $t \in(0,1) \backslash Q, p \in \mathbb{R}^{n}, r(t, x)=0$, and $r^{\prime}(t, x, p)=0$, then $r_{f}^{\prime \prime}(t, x, p)>0$. Since $f$ satisfies the Hartman-Nagumo condition, so $\|f\| \leq \Phi(\|p\|)$. It follows from (15) that

$$
M_{0} \Phi(\|p\|) r_{x} \cdot(x-v(t)) \geq\|f\|\left\|r_{x}\right\| \geq r_{x} \cdot f .
$$

If $\Phi(\|p\|) \leq L$ from (14), then $f_{0}=M_{0} \Phi(\|p\|)[x-v(t)]$ and

$$
r_{f_{0}}^{\prime \prime}=r_{0}^{\prime \prime}+r_{x} \cdot M_{0} \Phi(\|p\|)[x-v(t)] \geq r_{f}^{\prime \prime}>0 .
$$

If $L \leq \Phi(\|p\|)$ from (14), then $f_{0}=M_{0} L[x-v(t)]$. It follows from (16) and (7) that

$$
r_{f_{0}}^{\prime \prime}=r_{0}^{\prime \prime}+r_{x} \cdot M_{0} L[x-v(t)]>r_{0}^{\prime \prime}+K_{1}>0
$$

Thus

$$
\begin{aligned}
r_{f_{\lambda}}^{\prime \prime}(t, x, p) & =\lambda\left[r_{0}^{\prime \prime}+r_{x} \cdot f(t, x, p)\right]+(1-\lambda)\left[r_{0}^{\prime \prime}+r_{x} \cdot f_{0}(t, x, p)\right] \\
& =\lambda r_{f}^{\prime \prime}+(1-\lambda) r_{f_{0}}^{\prime \prime}>0 .
\end{aligned}
$$

Now we construct the second one-parameter family of systems of ordinary differential equations.

For $\lambda \in[0,1]$, we define $f_{1, \lambda}:[0,1] \times \mathbb{R}^{2 n} \rightarrow \mathbb{R}^{n}$ by

$$
f_{1, \lambda}(t, x, p)=\lambda f_{0}(t, x, p)+(1-\lambda) M_{0} L[x-v(t)],
$$

where $f_{0}, M_{0}, L$ are given in (14), (15), and (16), respectively.

We consider the system

$$
x^{\prime \prime}=f_{1, \lambda}\left(t, x, x^{\prime}\right) \quad \text { for all } t \in[0,1] \backslash Q \text {. }
$$


Lemma 3 Assume that $(\Omega, v)$ is an admissible bounding set for $(1)$ and that $f_{1, \lambda}$ is defined in (20). Then, for $(t, x, p) \in \bar{\Omega} \times \mathbb{R}^{n}$,

$$
\begin{aligned}
\left\|f_{1, \lambda}(t, x, p)\right\| & \leq M_{0} L R_{1} \quad \text { and } \\
\left\|f_{1, \lambda}(t, x, p)\right\| & \leq M_{2} r_{f_{1, \lambda}^{\prime \prime}}^{\prime \prime}(t, x, p)+K_{2},
\end{aligned}
$$

where $R_{1}$ is given in (10).

If $x$ is a solution of $(21)$ with $(t, x) \in \bar{\Omega}$, then $\left\|x^{\prime}(t)\right\|<N$, where $N$ is given in Lemma 1 .

Moreover, if $t \in(0,1) \backslash Q, p \in \mathbb{R}^{n}, r(t, x)=0$, and $r^{\prime}(t, x, p)=0$, then $r_{f_{1, \lambda}}^{\prime \prime}(t, x, p)>0$.

Proof Clearly,

$$
\left\|f_{1, \lambda}(t, x, p)\right\| \leq \lambda\left\|f_{0}(t, x, p)\right\|+(1-\lambda) \| M_{0} L\left(x-v(t) \| \leq M_{0} L R_{1}\right.
$$

for all $(t, x, p) \in \bar{\Omega} \times \mathbb{R}^{n}$, where $R_{1}$ is given in (10). From the proof of Lemma $2,\left\|f_{0}(t, x, p)\right\| \leq$ $M_{2} r_{f_{0}}^{\prime \prime}+K_{2}$ for all $(t, x) \in \bar{\Omega}, p \in \mathbb{R}^{n}$. It follows from (17) and (8) that

$$
\begin{aligned}
\left\|M_{0} L[x-v(t)]\right\| & \leq M_{2}\left\{r_{0}^{\prime \prime}+r_{x} \cdot M_{0} \min \{L, \Phi(\|p\|)\}[x-v(t)]\right\}+K_{2} \\
& \leq M_{2}\left\{r_{0}^{\prime \prime}+r_{x} \cdot M_{0} L(x-v(t))\right\}+K_{2} \\
& =M_{2} r_{M_{0} L(x-v(t))}^{\prime \prime}+K_{2} .
\end{aligned}
$$

Thus

$$
\begin{aligned}
\left\|f_{1, \lambda}(t, x, p)\right\| & \leq \lambda\left\|f_{0}(t, x, p)\right\|+(1-\lambda)\left\|M_{0} L(x-v(t))\right\| \\
& \leq \lambda\left[M_{2} r_{f_{0}}^{\prime \prime}+K_{2}\right]+(1-\lambda)\left[M_{2} r_{M_{0} L(x-v(t))}^{\prime \prime}+K_{2}\right] \\
& =M_{2} r_{f_{1, \lambda}^{\prime \prime}}^{\prime \prime}+K_{2} .
\end{aligned}
$$

If $x$ is a solution of (21) with $(t, x) \in \bar{\Omega}$, then it follows from Lemma 1 that $\left\|x^{\prime}(t)\right\|<N$, where $N$ is given in Lemma 1.

From the proof of Lemma 2, if $t \in(0,1) \backslash Q, p \in \mathbb{R}^{n}, r(t, x)=0, r^{\prime}(t, x, p)=0$, then $r_{f_{0}}^{\prime \prime}(t, x, p)>0$. It follows from (15) and (16), respectively, that

$$
r_{x} \cdot M_{0} L(x-v(t))>K_{1} \quad \text { for }(t, x) \in \partial_{C} \Omega,
$$

where $K_{1}$ is given in Remark 1(i). Therefore,

$$
\begin{aligned}
r_{f_{1, \lambda}}^{\prime \prime}(t, x, p) & =\lambda\left[r_{0}^{\prime \prime}+r_{x} \cdot f_{0}(t, x, p)\right]+(1-\lambda)\left[r_{0}^{\prime \prime}+r_{x} \cdot M_{0} L(x-v(t))\right] \\
& >\lambda r_{f_{0}}^{\prime \prime}(t, x, p)+(1-\lambda)\left[r_{0}^{\prime \prime}(t, x, p)+K_{1}\right] \\
& >0 .
\end{aligned}
$$

Remark 5 If $M=0$, where $M$ is given in Definition 2, we do not need to choose $M_{2}$ and $K_{2}$ in (14). We set

$$
f_{0}(t, x, p)=M_{0} L[x-v(t)]
$$


for $(t, x, p) \in[0,1] \times \mathbb{R}^{2 n}$. Moreover, we do not need the second one-parameter family of systems of ordinary differential equations based on $f_{1, \lambda}$ to construct our homotopy.

For $0 \leq k \leq q$ and $(t, x) \in \bar{\Omega}_{k}$, let $G_{k}: \bar{J}_{k} \times \bar{J}_{k} \rightarrow \mathbb{R}$ be Green's function for (1) restricted to $\bar{J}_{k}$ together with the homogeneous boundary conditions $x_{k}\left(t_{k}\right)=A=0=B=x_{k}\left(t_{k+1}\right)$, thus

$$
G_{k}(t, s)= \begin{cases}\frac{\left(t-t_{k}\right)\left(t_{k+1}-s\right)}{t_{k+1} t_{k}} & \text { for } t_{k} \leq t \leq s \leq t_{k+1}, \\ \frac{\left(t_{k+1}-t\right)\left(s-t_{k}\right)}{t_{k+1}-t_{k}} & \text { for } t_{k} \leq s \leq t \leq t_{k+1} .\end{cases}
$$

For $0 \leq k \leq q$, let

$$
w_{k}\left(C_{k}, D_{k+1}\right)(t)=\frac{\left(t_{k+1}-t\right) C_{k}}{t_{k+1}-t_{k}}+\frac{\left(t-t_{k}\right) D_{k+1}}{t_{k+1}-t_{k}} .
$$

Using the above two families of systems of ordinary differential equations, we can homotopy the original problem (1), (2), and (3) to the following solvable system of ordinary differential equations subject to Picard boundary conditions and impulses.

$$
\begin{aligned}
& x^{\prime \prime}=M_{0} L[x-v(t)] \quad \text { for } t \in[0,1] \backslash Q, \\
& x\left(t_{k}^{+}\right)=v\left(t_{k}^{+}\right) \quad \text { and } \quad x\left(t_{k+1}^{-}\right)=v\left(t_{k+1}^{-}\right), \quad \forall 0 \leq k \leq q,
\end{aligned}
$$

where $M_{0}, L$ are given in (15) and (16), respectively. Then (24) and (25) have a solution $V \in C_{Q}^{2}=C_{Q}^{2}[0,1]$ of the form

$$
\begin{aligned}
V(t)= & V_{k}(t) \\
= & \frac{\left(t_{k+1}-t\right) v\left(t_{k}^{+}\right)}{t_{k+1}-t_{k}}+\frac{\left(t-t_{k}\right) v\left(t_{k+1}^{-}\right)}{t_{k+1}-t_{k}} \\
& -M_{0} L \int_{t_{k}}^{t_{k+1}} G_{k}(t, s)\left[V_{k}(s)-v_{k}(s)\right] d s \\
= & w_{k}\left(v\left(t_{k}^{+}\right), v\left(t_{k+1}^{-}\right)\right)(t) \\
& -M_{0} L \int_{t_{k}}^{t_{k+1}} G_{k}(t, s)\left[V_{k}(s)-v_{k}(s)\right] d s
\end{aligned}
$$

for $t \in J_{k}$ and $0 \leq k \leq q$, where we have identified $V$ with $\tilde{V}=\left(V_{0}, \ldots, V_{q}\right)$.

We show that $V(t) \in \Omega(t)$ for all $t \in[0,1]$.

Lemma 4 Assume that $(\Omega, v)$ is an admissible bounding set for (1) and $V(t)$ is given by (26). Then $V(t) \in \Omega(t)$ for $t \in[0,1]$. Moreover, $\left\|V^{\prime}(t)\right\|<N$, where $N$ is given in Lemma 1.

Proof Suppose $(\tilde{t}, V(\tilde{t})) \notin \Omega$ for some $\tilde{t} \in \bar{J}_{k}$. Set $q(t)=[V(t)-v(t)]^{2}$. Since $V(t)$ is a solution of (24) and (25), it follows that $q\left(t_{k}^{+}\right)=q\left(t_{k+1}^{-}\right)=0, \forall 0 \leq k \leq q$, and so $\tilde{t} \neq t_{k}$ and $\tilde{t} \neq t_{k+1}$. Therefore $\tilde{t} \in J_{k}^{\circ}$. So $q(t)$ has a local maximum at $\tilde{t} \in J_{k}^{\circ}$ and $(\tilde{t}, V(\tilde{t})) \notin \Omega$. Hence $q(\tilde{t}) \geq \varepsilon^{2}$, where $\varepsilon$ is given below. But it follows from (15) and (16) that $M_{0} L \varepsilon>\left\|v^{\prime \prime}(t)\right\|$ for all $t \in[0,1]$ 
and hence

$$
\begin{aligned}
\frac{q^{\prime \prime}(\tilde{t})}{2} & =\left[V^{\prime}(\tilde{t})-v^{\prime}(\tilde{t})\right]^{2}+[V(\tilde{t})-v(\tilde{t})]^{2} M_{0} L-[V(\tilde{t})-v(\tilde{t})] v^{\prime \prime}(\tilde{t}) \\
& \geq\left[V^{\prime}(\tilde{t})-v^{\prime}(\tilde{t})\right]^{2}+\|V(\tilde{t})-v(\tilde{t})\|\left[\varepsilon M_{0} L-\left\|v^{\prime \prime}(\tilde{t})\right\|\right] \\
& >0
\end{aligned}
$$

a contradiction. Thus $(t, V(t)) \in \Omega$ for $t \in[0,1]$. Since $V(t)$ is a solution of (24) and (21) is (24) when $\lambda=0$, it follows from Lemma 3 that $\left\|V^{\prime}(t)\right\|<N$, where $N$ is given in Lemma 1 .

Now we present our main result.

Theorem 1 Assume that $(\Omega, v)$ is an admissible bounding set for $(1)$ and that $f$ satisfies the Hartman-Nagumo condition. Suppose that the boundary conditions (2) and impulses (3) are compatible with $\Omega$. Then there is at least one solution $x \in C_{Q}^{2}$ of problem (1), (2), and (3) $\operatorname{such}$ that $(t, x(t)) \in \bar{\Omega}$ for $t \in[0,1]$.

Proof Now $\Delta_{k} \neq \emptyset$ for $0 \leq k \leq q$. First consider the case that all $g_{k}$ are strongly compatible with $\Omega$.

Choose $\varepsilon \in(0,1)$ such that $B_{\varepsilon}(v(t)) \subseteq \Omega(t)$ for all $t \in[0,1]$. It follows from Remark 1(ii) that $(x-v(t)) \cdot r_{x}(t, x)>\eta c>0$, where $\eta>0, c>0$, for $x \in \bigcup_{k} \partial_{C} \Omega_{k}(t)$ and all $t \in[0,1]$. Let $M_{0}, L$, and $K_{2}$ be given in (15), (16), and (17), respectively. Let $M_{2}>M$ where $M$ is given in Definition 2.

Let

$$
\Pi=\left\{x \in C_{Q}^{1}:\left(t, x_{k}(t)\right) \in \Omega_{k},\left\|x_{k}^{\prime}(t)\right\|<N, \forall t \in \bar{J}_{k}, 0 \leq k \leq q\right\},
$$

and let $\Sigma=\Pi \times \Delta$, where $\Delta=\prod_{k=0}^{q} \Delta_{k}$ and $\Delta_{k}$ is given in (9) and $N$ is given in Lemma 1 .

Following [4], we interpret $(C, D)=\left(C_{0}, \ldots, C_{q}, D_{0}, \ldots D_{q}\right) \in \Delta$ to mean $\left(C_{k}, D_{k}\right) \in \Delta_{k}$ for $k=0, \ldots, q$ and set $D_{q+1}=D_{0}$. Let $\Psi(C, D)=\left(\Psi_{0}\left(C_{0}, D_{0}\right), \ldots, \Psi_{q}\left(C_{q}, D_{q}\right)\right)$, where $\Psi_{k}$ is a strongly inwardly pointing vector field on $\Delta_{k}$ for each $k$. Let $\mathcal{G}(C, D)=\left(\mathcal{G}_{0}\left(C_{0}, D_{0}\right), \ldots\right.$, $\left.\mathcal{G}_{q}\left(C_{q}, D_{q}\right)\right)$, where $\mathcal{G}_{k}\left(C_{k}, D_{k}\right)$ is given in (11), for all $0 \leq k \leq q$.

Let $f_{\lambda}$ be given in (18). For all $t \in \bar{J}_{k}$, let

$$
T_{k}\left(f_{\lambda}\left(x_{k}\right)\right)(t)=-\int_{t_{k}}^{t_{k+1}} G_{k}(t, s) f_{\lambda}\left(s, x_{k}(s), x_{k}^{\prime}(s)\right) d s,
$$

where $G_{k}(t, s)$ is given in (22). Define

$$
T\left(f_{\lambda}(x)\right)(t)=\left(T_{0}\left(f_{\lambda}\left(x_{0}\right)\right)(t), \ldots, T_{q}\left(f_{\lambda}\left(x_{q}\right)\right)(t)\right),
$$

where we identify $x$ and $\tilde{x}=\left(x_{0}, \ldots, x_{q}\right)$.

Consider the solutions $(x, C, D) \in \bar{\Sigma}$ of

$$
\Phi_{2}(x, C, D)=\left(x-T\left(f_{1}\right)-w(C, D), g\left(C, D, x^{\prime}(0), x^{\prime}(1)\right)\right)=(0,0),
$$


where

$$
w(C, D)(t)=\left(w_{0}\left(C_{0}, D_{1}\right)(t), \ldots, w_{q-1}\left(C_{q-1}, D_{q}\right)(t), w_{q}\left(C_{q}, D_{0}\right)(t)\right)
$$

and $w_{k}\left(C_{k}, D_{k+1}\right)(t)$ is given in (23) for all $0 \leq k \leq q$.

From (18) and (28), problem (1), (2), and (3) has a solution $x$ satisfying $(t, x) \in \bar{\Omega}$ if and only if $(x, C, D)$ is a solution of (29) in $\bar{\Sigma}$ since $C_{k}=x_{k}\left(t_{k}\right)$ and $D_{k}=x_{k-1}\left(t_{k}\right)$ for $0 \leq k \leq q$ in that case.

To show that (29) has a solution, we use Leray-Schauder degree theory.

Define $\mathcal{H}_{i}:[0,1] \times \bar{\Sigma} \rightarrow \prod_{k=0}^{q} C^{1}\left(\bar{J}_{k} ; \mathbb{R}^{n}\right) \times \mathbb{R}^{2(q+1) n}$ for $i=1,2,3$ by

$$
\begin{aligned}
\mathcal{H}_{1}(\lambda,(x, C, D))= & \left(x-T\left(f_{1}(x)\right)-w(C, D), \mathcal{S}(x, C, D, \lambda)\right) \\
\mathcal{H}_{2}(\lambda,(x, C, D))= & \left(x-T\left(f_{\lambda}(x)\right)\right. \\
& -w(\lambda C+(1-\lambda) v(0), \lambda D+(1-\lambda) v(1)), \mathcal{G}(C, D)) \\
\mathcal{H}_{3}(\lambda,(x, C, D))= & \left(x-T\left(f_{1, \lambda}(x)\right)-w(v(0), v(1)), \mathcal{G}(C, D)\right),
\end{aligned}
$$

where

$$
\begin{aligned}
& \mathcal{S}(x, C, D, \lambda)=g\left((C, D) ; \lambda\left(x^{\prime}(0), x^{\prime}(1)\right)+(1-\lambda) \Psi(C, D)\right) \text { and } \\
& g(C, D, u, v):=\left(g_{0}\left(C_{0}, D_{0}, u_{0}, v_{0}\right), \ldots, g_{q}\left(C_{q}, D_{q}, u_{q}, v_{q}\right)\right)
\end{aligned}
$$

for $(u, v)=\left(u_{0}, \ldots, u_{q}, v_{0}, \ldots v_{q}\right) \in \mathbb{R}^{2(q+1) n}, f_{1, \lambda}$ is given in $(20)$.

Now $\mathcal{H}_{i}$ is completely continuous since $T$ is completely continuous. We show that either there is a solution to our problem or the above functions $\mathcal{H}_{i}$ define homotopies.

It is easy to see that $(x, C, D) \in \bar{\Sigma}$ is a solution of (1), (2), and (3) with $(C, D)=$ $\left(x_{0}(0), x_{1}\left(t_{1}\right), \ldots, x_{q}\left(t_{q}\right), x_{0}\left(t_{1}\right), \ldots, x_{q}(1)\right) \in \bar{\Delta}$ if

$$
\mathcal{H}_{1}(x, C, D, \lambda)=0
$$

when $\lambda=1$. Now if there is a solution of (30) with $(x, C, D) \in \partial \Sigma$ for $\lambda=1$, then $(C, D)=$ $\left(x_{0}(0), x_{1}\left(t_{1}\right), \ldots, x_{q}\left(t_{q}\right), x_{0}\left(t_{1}\right), \ldots, x_{q}(1)\right) \in \bar{\Delta}$ and $x=\left(x_{0}, \ldots, x_{1}\right)$ is the required solution, so we assume there is no solution on $\partial \Sigma$. We show that $\mathcal{H}_{1}$ is a homotopy for the LeraySchauder degree on $\Sigma$ at 0 , that is, there are no solutions $(x, C, D) \in \partial \Sigma$ of (30) for $0 \leq$ $\lambda<1$. We argue by contradiction and assume that there is a solution of $(30)$ with $\lambda \in[0,1)$ and $(x, C, D) \in \partial \Sigma$. From the definition of $\mathcal{H}_{1}, x$ is a solution of (1) such that

$$
g\left((C, D) ; \lambda\left(x^{\prime}(0), x^{\prime}(1)\right)+(1-\lambda) \Psi(C, D)\right)=0 \in \mathbb{R}^{2(q+1) n}
$$

for $\lambda \in[0,1)$. Suppose $(C, D) \in \partial \triangle$. Assume $C_{k} \in \partial \Omega_{k}\left(t_{k}\right)$. Since $x_{k}\left(t_{k}\right)=C_{k}$, then $r_{k}\left(t_{k}, C_{k}\right)=0$ so that $r_{k}^{\prime}\left(t_{k}, C_{k}, x_{k}^{\prime}\left(t_{k}\right)\right) \leq 0$. Since $\Psi_{k}$ is a strongly inwardly pointing vector field on $\Delta_{k}$ for each $k$ and $0 \leq \lambda<1$, thus

$$
r_{k}^{\prime}\left(t_{k}, C_{k}, \lambda x_{k}^{\prime}\left(t_{k}\right)+(1-\lambda) \psi_{k}^{0}\left(C_{k}, D_{k}\right)\right)<0 .
$$


Thus $g_{k}\left(\left(C_{k}, D_{k}\right) ; \lambda\left(x_{k}^{\prime}\left(t_{k}\right), x_{k-1}^{\prime}\left(t_{k}\right)\right)+(1-\lambda) \Psi_{k}\left(C_{k}, D_{k}\right)\right) \neq 0$ as $g$ is strongly compatible with $\Omega$. Thus $\mathcal{S}(x, C, D, \lambda) \neq 0$, a contradiction. Similarly, the other cases $(C, D) \in \partial \triangle$ lead to a contradiction, so $(C, D) \notin \partial \triangle$. Suppose $x \in \partial \Pi$. By the choice of $N,\left\|x_{k}^{\prime}(t)\right\|<N$ for all $k$. Assume that $x(\tilde{t}) \in \partial \Omega(\tilde{t})$ for some $\tilde{t} \in J_{k}^{\circ}$. Then $r(\tilde{t}, x(\tilde{t}))=0$. Since $(t, x(t)) \in \bar{\Omega}$ for $t \in J_{k}^{\circ}$, it follows that $r$ attains a local maximum at $\tilde{t} \in J_{k}^{\circ}$. Thus $r^{\prime}\left(\tilde{t}, x(\tilde{t}), x^{\prime}(\tilde{t})\right)=0$. However, $r_{f}^{\prime \prime}\left(\tilde{t}, x(\tilde{t}), x^{\prime}(\tilde{t})\right)>0$, a contradiction. Thus $\mathcal{H}_{1}(\lambda,(x, C, D)) \neq 0$ for any $(x, C, D) \in \partial \Sigma$, $\lambda \in[0,1)$.

Suppose that $\mathcal{H}_{2}(\lambda,(x, C, D))=0$ has a solution $(x, C, D) \in \partial \Sigma$. From the definition of $\mathcal{H}_{2}$, $x$ is a solution of $(19)$ with $\mathcal{G}(C, D)=0$. Since $\mathcal{G}(C, D) \neq 0$ on $\partial \triangle$, it follows that $(C, D) \notin \partial \triangle$. Suppose $x \in \partial \Pi$. By the choice of $N,\left\|x_{k}^{\prime}(t)\right\|<N$ for all $k$. Assume that $x(\tilde{t}) \in \partial \Omega(\tilde{t})$ for some $\tilde{t} \in J_{k}^{\circ}$. Then $r(\tilde{t}, x(\tilde{t}))=0$. Since $(t, x(t)) \in \bar{\Omega}$ for $t \in J_{k}^{\circ}$, it follows that $r$ attains a local maximum at $\tilde{t} \in J_{k}^{\circ}$. Thus $r^{\prime}\left(\tilde{t}, x(\tilde{t}), x^{\prime}(\tilde{t})\right)=0$. However, $r_{f_{\lambda}}^{\prime \prime}\left(\tilde{t}, x(\tilde{t}), x^{\prime}(\tilde{t})\right)>0$ by Lemma 2 . Since $\left(C_{k}, D_{k}\right) \notin \partial \Delta_{k}$ and $B_{\varepsilon}(v(t)) \subseteq \Omega(t)$ for all $t \in[0,1]$, so $\left(v_{k}\left(t_{k}\right), v_{k-1}\left(t_{k}\right)\right) \in \Delta_{k}^{\circ}$ for $0 \leq$ $k \leq q$. Moreover, from Remark $1(\mathrm{i}), \Omega(t)$ is convex for all $t \in[0,1]$, it follows that $x_{k}\left(t_{k}\right)=$ $\lambda C_{k}+(1-\lambda) v_{k}\left(t_{k}\right) \notin \partial \Omega_{k}\left(t_{k}\right)$ and $x_{k-1}\left(t_{k}\right)=\lambda D_{k}+(1-\lambda) v_{k-1}\left(t_{k}\right) \notin \partial \Omega_{k-1}\left(t_{k}\right)$ for all $k=$ $0, \ldots, q$. Thus $x \notin \bigcup_{k} \partial_{C} \Omega_{k}$. Therefore $\mathcal{H}_{2}(\lambda,(x, C, D)) \neq 0$ for any $(x, C, D) \in \partial \Sigma, \lambda \in[0,1]$.

Suppose that $\mathcal{H}_{3}(\lambda,(x, C, D))=0$ has a solution $(x, C, D) \in \partial \Sigma$. From the definition of $\mathcal{H}_{3}$, $x$ is a solution of (21) with $x_{k}\left(t_{k}\right)=v_{k}\left(t_{k}\right) \notin \partial \Omega_{k}\left(t_{k}\right)$ and $x_{k-1}\left(t_{k}\right)=v_{k-1}\left(t_{k}\right) \notin \partial \Omega_{k-1}\left(t_{k}\right)$ for all $0 \leq k \leq q$ and $\mathcal{G}(C, D)=0$. Since $\mathcal{G}(C, D) \neq 0$ on $\partial \Delta$, so $(C, D) \notin \partial \triangle$. Suppose $x \in \partial \Pi$. By the choice of $N,\left\|x_{k}^{\prime}(t)\right\|<N$ for all $k$. Assume that $x(\tilde{t}) \in \partial \Omega(\tilde{t})$ for some $\tilde{t} \in J_{k}^{\circ}$. Then $r(\tilde{t}, x(\tilde{t}))=0$. Since $(t, x(t)) \in \bar{\Omega}$ for $t \in J_{k}^{\circ}$, it follows that $r$ attains a local maximum at $\tilde{t} \in J_{k}^{\circ}$. Thus $r^{\prime}\left(\tilde{t}, x(\tilde{t}), x^{\prime}(\tilde{t})\right)=0$. However, $r_{f_{1, \lambda}}^{\prime \prime}\left(\tilde{t}, x(\tilde{t}), x^{\prime}(\tilde{t})\right)>0$ by Lemma 3 , so $x \notin \bigcup_{k} \partial_{C} \Omega_{k}$. Thus $\mathcal{H}_{3}(\lambda,(x, C, D)) \neq 0$ for any $(x, C, D) \in \partial \Sigma, \lambda \in[0,1]$.

Therefore $\mathcal{H}_{i}$ are homotopies for $i=1,2,3$. For all $\lambda \in[0,1]$ and $i=1,2,3$, by the homotopy invariance of the Leray-Schauder degree, we have

$$
d\left(\mathcal{H}_{i}(\lambda, \cdot), \Sigma, 0\right)=\text { constant }
$$

In particular,

$$
\begin{aligned}
d\left(\Phi_{2}, \Sigma, 0\right) & =d\left(\mathcal{H}_{1}(1, \cdot), \Sigma, 0\right)=d\left(\mathcal{H}_{2}(\lambda, \cdot), \Sigma, 0\right) \\
& =d\left(\mathcal{H}_{3}(\lambda, \cdot), \Sigma, 0\right)=d\left(\mathcal{H}_{3}(0, \cdot), \Sigma, 0\right) \\
& =d\left(\mathcal{I}-M_{0} L T, \Pi, W\right) \cdot d(\mathcal{G}, \Delta, 0) \\
& \in\{d(\mathcal{G}, \Delta, 0),-d(\mathcal{G}, \Delta, 0)\} \\
& =\left\{\prod_{k=0}^{q} d\left(\mathcal{G}_{k}, \Delta_{k}, 0\right),-\prod_{k=0}^{q} d\left(\mathcal{G}_{k}, \Delta_{k}, 0\right)\right\} \\
& \neq 0,
\end{aligned}
$$

where $T$ is defined in (28) and $W$ is given by

$$
W(t)=w_{k}\left(v\left(t_{k}^{+}\right), v\left(t_{k+1}^{-}\right)\right)(t)+M_{0} L \int_{t_{k}}^{t_{k+1}} G_{k}(t, s) v_{k}(s) d s
$$

for $t \in\left(t_{k}, t_{k+1}\right)$, where $w_{k}\left(v\left(t_{k}^{+}\right), v\left(t_{k+1}^{-}\right)\right)(t)$ and $G_{k}(t, s)$ are given in (23) and (22), respectively. Moreover, since $V \in \Pi$ is the solution of (26), using the Leray index theorem, The- 
orem 8.10 in [23], it is easy to show

$$
\begin{aligned}
d\left(\mathcal{I}-M_{0} L T, \Pi, W\right) & =d\left(\mathcal{I}-M_{0} L T, \Pi-V, 0\right) \\
& =d\left(\mathcal{I}-M_{0} L T, B_{1}, 0\right) \in\{1,-1\},
\end{aligned}
$$

where $B_{1}$ is an open ball in $\Pi-V=\{x: x+V \in \Pi\}$. Thus there is a solution $(x, C, D) \in \Sigma$ of $\mathcal{H}_{1}(1,(x, C, D))=0$ and $x \in C_{Q}^{1}$ is a solution of (29). By the above argument, $x$ is the required solution of (1), (2), and (3).

Suppose now that $g_{k}$ for $0 \leq k \leq q$ is compatible with $\Omega_{k}$. Then there is a sequence $\left\{g_{k_{i}}\right\}_{i=1}^{\infty}$ strongly compatible with $\Omega_{k}$ and converging uniformly to $g_{k}$ on compact subsets of $\bar{\Delta}_{k} \times \mathbb{R}^{2 n}$ for $0 \leq k \leq q$. Let $y_{i}$ be the corresponding solution. By compactness, there is a subsequence of $y_{i_{j}}$ converging in $C_{Q}^{1}$ to the desired solution of integral equation (29), and hence the differential equation, satisfying the boundary conditions and impulses.

\section{Remark 6}

(i) It is easy to see from the above proof that we can weaken our assumptions as follows. We assume that $f \in C\left(\{[0,1] \backslash Q\} \times \mathbb{R}^{n} ; \mathbb{R}^{n}\right)$ and look for solutions $x \in C_{Q}^{1} \cap C^{2}\left(\{[0,1] \backslash Q\} \times \mathbb{R}^{n} ; \mathbb{R}^{n}\right)$. Moreover, we may assume that $\left.r\right|_{J_{k}^{\circ}} \in C^{2}\left(J_{k}^{\circ} \times \mathbb{R}^{n}\right)$ and has an extension $r_{k} \in C^{1}\left(\bar{J}_{k} \times \mathbb{R}^{n}\right)$.

(ii) We can vary the assumptions on our admissible bounding sets. It is easy to see from the proof that instead of assuming that $p^{T} r_{x x}(t, x) p \geq \Theta\|p\|^{2}$ for some constants $\Theta>0, p \in \mathbb{R}^{n}$, and $(t, x) \in \Omega$, it suffices to assume that $p^{T} r_{x x}(t, x) p+2 p^{T} r_{t x}(t, x) \geq 0$ for $p \in \mathbb{R}^{n}$ and $(t, x) \in \Omega$. Indeed, we can still recover our existence result by an approximation argument if we can weaken this further to $p^{T} r_{x x}(t, x) p \geq 0$ for $p \in \mathbb{R}^{n}$ and $(t, x) \in \Omega$. We apply our Theorem using $r_{\varepsilon}=r+\varepsilon \frac{x^{2}}{2}$ noting that $r_{\varepsilon, x x}$ satisfies (6) and $\Omega_{\varepsilon}=\left\{(t, x) \in[0,1] \times \mathbb{R}^{n}: r_{\varepsilon}(t, x)<0\right\} \subseteq \Omega$. Since solutions $x_{\varepsilon}$ with $\left(t, x_{\varepsilon}(t)\right) \in \bar{\Omega}_{\varepsilon}$ satisfy $\left(t, x_{\varepsilon}(t)\right) \in \bar{\Omega}$, we obtain derivative bounds independent of $\varepsilon$. Since $\left\|r_{x}\right\| \neq 0$ on $\bigcup_{k} \partial_{C} \Omega_{k}$, strongly compatible boundary conditions on $\Omega$ will be strongly compatible on $\Omega_{\varepsilon}$ for $0<\varepsilon$ sufficiently small. Letting $\varepsilon$ approach 0 and choosing a subsequence if necessary, $x_{\varepsilon}$ converges to a solution of our problem.

\section{Example}

In this section we present an example to illustrate the power of our existence result. This example is modeled on that in [2] and we have added impulses.

Example 1 Let $x=\left(x_{1}, x_{2}\right)$ and $f=\left(f_{1}, f_{2}\right)$ and consider the problem

$$
\begin{aligned}
x_{1}^{\prime \prime} & =2 x_{1}+\sin 2 \pi t-x_{1} x_{2}^{3}-w\left(t, x_{1}, x_{2}, x_{1}^{\prime}, x_{2}^{\prime}\right) x_{2}^{3} x_{1}^{\prime}+\frac{x_{1}^{\prime 2}}{4}+x_{2}^{2} x_{2}^{\prime 2} \\
& =f_{1}\left(t, x, x^{\prime}\right), \\
x_{2}^{\prime \prime} & =x_{2}+\cos 2 \pi t+x_{1}^{2}+w\left(t, x_{1}, x_{2}, x_{1}^{\prime}, x_{2}^{\prime}\right) x_{1} x_{1}^{\prime} \\
& =f_{2}\left(t, x, x^{\prime}\right)
\end{aligned}
$$


for $t \in[0,1] \backslash\{1 / 2\}$, where $w$ is a bounded continuous function. Let $\Omega=\Omega_{0} \cup \Omega_{1}$, where

$$
\begin{aligned}
& \Omega_{0}=\left\{(t, x) \in\left[0, \frac{1}{2}\right] \times \mathbb{R}^{2}: r_{0}(t, x)=\frac{x_{1}^{2}}{2}+\frac{x_{2}^{4}}{4}-\frac{1}{2}<0\right\}, \\
& \Omega_{1}=\left\{(t, x) \in\left(\frac{1}{2}, 1\right] \times \mathbb{R}^{2}: r_{1}(t, x)=\frac{x_{1}^{2}}{2}+\frac{x_{2}^{4}}{4}-2<0\right\} .
\end{aligned}
$$

Let $v(t)=(0,0)$ for all $t \in[0,1]$, and let the Sturm-Liouville boundary conditions be given by

$$
x^{\prime}(0)=x(0), \quad x^{\prime}(1)=-x(1)
$$

Let the impulses be given by

$$
\begin{aligned}
& 2 x_{1}\left(\frac{1^{-}}{2}\right)=x_{1}\left(\frac{1}{2}^{+}\right), \quad \sqrt{2} x_{2}\left(\frac{1}{2}^{-}\right)=x_{2}\left(\frac{1}{2}^{+}\right), \\
& x_{1}^{\prime}\left(\frac{1^{-}}{2}\right)-x_{1}^{\prime}\left(\frac{1^{+}}{2}\right)+\delta x_{1}\left(\frac{1}{2}^{-}\right)\left[1+\left|x_{1}\left(\frac{1^{-}}{2}\right)\right|^{2}\right]=0, \\
& x_{2}^{\prime}\left(\frac{1}{2}^{-}\right)-\sqrt{2} x_{2}^{\prime}\left(\frac{1^{+}}{2}\right)+\delta x_{2}\left(\frac{1}{2}^{-}\right)\left[1+\left|x_{2}\left(\frac{1^{-}}{2}\right)\right|^{2}\right]=0,
\end{aligned}
$$

where $\delta>0$.

To see that $(\Omega, v)$ is an admissible bounding set, first we note $r^{\prime}(t, x, p)=x_{1} p_{1}+x_{2}^{3} p_{2}$ and

$$
\begin{aligned}
r_{f}^{\prime \prime}(t, x, p)= & x_{1}\left(2 x_{1}+\sin 2 \pi t\right)+x_{2}^{3}\left(x_{2}+\cos 2 \pi t\right) \\
& +\left(\frac{x_{1}}{4}+1\right) p_{1}^{2}+\left(x_{1} x_{2}^{2} p_{2}^{2}+3 x_{2}^{2} p_{2}^{2}\right),
\end{aligned}
$$

for $t \in(0,1) \backslash\{1 / 2\}$. If $t \in(0,1 / 2), r(t, x)=0$ and $r^{\prime}(t, x, p)=0$, then Kongson et al. [2] proved that $r_{f}^{\prime \prime}>0$ for $(t, x) \in \partial_{C} \Omega_{0}$. We prove $r_{f}^{\prime \prime}>0$ for $(t, x) \in \partial_{C} \Omega_{1}$.

Now $\left(\frac{x_{1}}{4}+1\right) p_{1}^{2}+\left(x_{1} x_{2}^{2} p_{2}^{2}+3 x_{2}^{2} p_{2}^{2}\right) \geq \frac{\left(p_{1}^{2}+x_{2}^{2} p_{2}^{2}\right)}{2} \geq 0$ for all $(t, x, p) \in \bar{\Omega}_{1} \times \mathbb{R}^{2}$ since $\left\|x_{1}\right\| \leq 2$, $\left\|x_{2}\right\| \leq 8^{\frac{1}{4}}$. Moreover, it is not difficult to show that

$$
\begin{aligned}
& 2 x_{1}^{2}+x_{1} \sin 2 \pi t+x_{2}^{4}+x_{2}^{3} \cos 2 \pi t \\
& \quad \geq 2\left(\frac{x_{1}^{2}}{2}+\frac{x_{2}^{4}}{4}\right)-\left[-\frac{x_{2}^{4}}{2}+\frac{x_{2}^{6}}{4}+\left(\sin ^{2} 2 \pi t+\cos ^{2} 2 \pi t\right)\right]>0
\end{aligned}
$$

for $(t, x) \in \partial_{C} \Omega_{1}$. Thus $r_{f}^{\prime \prime}(t, x, p)>0$ for $(t, x) \in \partial_{C} \Omega_{1}$.

It is not difficult to prove that $f$ satisfies the Hartman-Nagumo condition. Some details are similar to those in the analysis of Example 1 given in Kongson et al. [2].

To show that the impulses given in (33) are compatible with $\Omega$, let $\Psi_{1}\left(C_{1}, D_{1}\right)=$ $\left(\psi_{1}^{0}\left(C_{1}, D_{1}\right), \psi_{1}^{1}\left(C_{1}, D_{1}\right)\right)=\left(\left(-C_{1,1},-C_{1,2}\right),\left(D_{1,1}, D_{1,2}\right)\right)$ be a strongly inwardly pointing vector field on $\Delta_{1}=\Omega\left(\frac{1}{2}^{+}\right) \times \Omega\left(\frac{1}{2}^{-}\right)$. Then

$$
\begin{aligned}
& r_{1}^{\prime}\left(\frac{1}{2}, C_{1}, \psi_{1}^{0}\left(C_{1}, D_{1}\right)\right)<0 \quad \text { for } C_{1} \in \partial \Omega\left(\frac{1}{2}^{+}\right), \\
& r_{0}^{\prime}\left(\frac{1}{2}, D_{1}, \psi_{1}^{1}\left(C_{1}, D_{1}\right)\right)>0 \quad \text { for } D_{1} \in \partial \Omega\left(\frac{1}{2}^{-}\right) .
\end{aligned}
$$


Let $g_{1}=\left(g_{1,1}, g_{1,2}\right)$ be given by

$$
\begin{aligned}
& g_{1,1}\left(C_{1}, D_{1}\right)=\left(2 D_{1,1}-C_{1,1}, \sqrt{2} D_{1,2}-C_{1,2}\right)=0, \\
& g_{1,2}\left(C_{1}, D_{1}, u_{1}, v_{1}\right)=\left(v_{1,1}-u_{1,1}+\delta D_{1,1}\left[1+\left|D_{1,1}\right|^{2}\right],\right. \\
& \left.v_{1,2}-\sqrt{2} u_{1,2}+\delta D_{1,2}\left[1+\left|D_{1,2}\right|^{2}\right]\right)=0
\end{aligned}
$$

for $\left(C_{1}, D_{1}, u_{1}, v_{1}\right) \in \bar{\Delta}_{1} \times \mathbb{R}^{2}$ so that the boundary conditions (33) are given by $g_{1}\left(x\left(\frac{1}{2}{ }^{+}\right)\right.$, $\left.x\left(\frac{1}{2}^{-}\right), x^{\prime}\left(\frac{1}{2}^{+}\right), x^{\prime}\left(\frac{1}{2}^{-}\right)\right)=0$. Therefore

$$
\begin{aligned}
\mathcal{G}_{1}\left(C_{1}, D_{1}\right)= & \left(g_{1,1}, g_{1,2}\right)\left(C_{1}, D_{1}, \psi_{1}^{0}\left(C_{1}, D_{1}\right), \psi_{1}^{1}\left(C_{1}, D_{1}\right)\right) \\
= & \left(\left(2 D_{1,1}-C_{1,1}, \sqrt{2} D_{1,2}-C_{1,2}\right),\left(\left(1+\delta\left[1+\left|D_{1,1}\right|^{2}\right]\right) D_{1,1}\right.\right. \\
& \left.\left.+C_{1,1},\left(1+\delta\left[1+\left|D_{1,2}\right|^{2}\right]\right) D_{1,2}+\sqrt{2} C_{1,2}\right)\right),
\end{aligned}
$$

and so

$$
\mathcal{G}_{1}\left(C_{1}, D_{1}\right) \cdot\left(\left(-\frac{1}{2} C_{1,1},-C_{1,2}\right),\left(D_{1,1}, D_{1,2}\right)\right)>0
$$

for $\left(C_{1}, D_{1}\right) \in \partial \Delta_{1}$. Thus

$$
\mathcal{H}_{1}\left(\lambda, C_{1}, D_{1}\right)=\lambda \mathcal{G}_{1}\left(C_{1}, D_{1}\right)+(1-\lambda)\left(\left(-\frac{1}{2} C_{1,1},-C_{1,2}\right),\left(D_{1,1}, D_{1,2}\right)\right)
$$

is a homotopy for the Brouwer degree and

$$
\begin{aligned}
d\left(\mathcal{G}_{1}, \Delta_{1}, 0\right) & =d\left(\mathcal{H}_{1}(1, \cdot), \Delta_{1}, 0\right)=d\left(\mathcal{H}_{1}(0, \cdot), \Delta_{1}, 0\right) \\
& =d\left(\mathcal{H}_{1,1}(0, \cdot), \Omega\left(\frac{1}{2}^{+}\right), 0\right) d\left(\mathcal{H}_{1,2}(0, \cdot), \Omega\left(\frac{1}{2}^{-}\right), 0\right) \\
& =(-1)^{2}=1 .
\end{aligned}
$$

Therefore, the impulses are strongly compatible with $\Omega$ and hence compatible. Using a similar proof, we can show that the boundary conditions given in (32) are strongly compatible with $\Omega$ and hence compatible.

Therefore our impulsive boundary value problem satisfies the conditions of Remark 6 (ii) and therefore has a solution $x \in C_{\{1 / 2\}}^{2}$ with $x(t) \in \Omega(t)$ for all $t \in[0,1]$.

\section{Competing interests}

The authors declare that they have no competing interests.

\section{Authors' contributions}

Both authors participated in the essential technical work of this article and read and approved the final manuscript. The authors contributed to this work equally.

\section{Acknowledgements}

The first author thanks the University of Queensland for University of Queensland International Scholarship (UQI) and University of Queensland Research Scholarship (UQRS). 


\section{References}

1. Thompson, HB: Systems of differential equations with fully nonlinear boundary conditions. Bull. Aust. Math. Soc. 56 197-208 (1997)

2. Kongson, J, Thompson, HB, Lenbury, Y: Multiple solutions for systems of differential equations with nonlinear boundary conditions. Nonlinear Stud. 18, 27-50 (2011)

3. Schmitt, K, Thompson, R: Boundary value problems for infinite systems of second-order differential equations. J. Differ. Equ. 18, 277-295 (1975)

4. Cabada, A, Thompson, HB: Nonlinear second-order equations with functional implicit impulses and nonlinear functional boundary conditions. Nonlinear Anal. 74, 7198-7209 (2011)

5. Benchohra, M, Henderson, J, Ntouyas, SK: On a periodic boundary value problem for first order impulsive differential inclusions. Dyn. Syst. Appl. 10, 477-488 (2001)

6. Shen, J: New maximum principles for first-order impulsive boundary value problems. Appl. Math. Lett. 16, 105-112 (2003)

7. Rachunková, I, Tvrdý, M: Impulsive periodic boundary value problem and topological degree. Funct. Differ. Equ. 9 471-498 (2002)

8. Rachunková, I, Tvrdý, M: Existence results for impulsive second-order periodic problems. Nonlinear Anal. 59, 133-146 (2004)

9. Rachunková, I, Tvrdý, M: Non-ordered lower and upper functions in second order impulsive periodic problems. Dyn. Contin. Discrete Impuls. Syst., Ser. A Math. Anal. 12, 397-415 (2005)

10. Cabada, A, Liz, E, Lois, S: Green's function and maximum principle for higher order ordinary differential equations with impulses. Rocky Mt. J. Math. 30, 435-446 (2000)

11. Liu, Y, Yang, P, Ge, W: Solutions of two-point BVPs at resonance for higher order impulsive differential equations. Nonlinear Anal. 60, 887-923 (2005)

12. Cabada, A, Liz, E: Discontinuous impulsive differential equations with nonlinear boundary conditions. Nonlinear Anal. 28, 1491-1497 (1997)

13. Erbe, LH, Liu, X: Existence results for boundary value problems of second order impulsive differential equations. J. Math. Anal. Appl. 149, 56-69 (1990)

14. Liz, E: Existence and approximation of solutions for impulsive first order problems with nonlinear boundary conditions. Nonlinear Anal. 25, 1191-1198 (1995)

15. Rachunková, I, Tomeček, J: Impulsive BVPs with nonlinear boundary conditions for the second order differential equations without growth restrictions. J. Math. Anal. Appl. 292, 525-539 (2004)

16. Polášek, V Periodic BVP with $\phi$-Laplacian and impulses. Acta Univ. Palacki. Olomuc., Fac. Rerum Nat., Math. 44 $131-150(2005)$

17. Cabada, A, Tomeček, J: Extremal solutions for nonlinear functional $\phi$-Laplacian impulsive equations. Nonlinear Anal. 67, 827-841 (2007)

18. Vial, J: Strong convexity of sets and functions. J. Math. Econ. 9, 187-205 (1982)

19. Evans, LC: Partial Differential Equation. Am. Math. Soc., Providence (2010)

20. Mawhin, J, Ureña, AJ: A Hartman-Nagumo inequality for the vector ordinary p-Laplacian and applications to nonlinear boundary value problems. J. Inequal. Appl. 7, 701-725 (2002)

21. Thompson, HB: Second order ordinary differential equations with fully nonlinear two point boundary conditions. Pac J. Math. 172(255-277), 279-297 (1996)

22. Hartman, P: Ordinary Differential Equations. Wiley, New York (1964)

23. Deimling, K: Nonlinear Functional Analysis. Springer, Berlin (1985)

10.1186/1687-2770-2013-240

Cite this article as: Song and Thompson: Systems of differential equations with implicit impulses and fully nonlinear boundary conditions. Boundary Value Problems 2013, 2013:240

\section{Submit your manuscript to a SpringerOpen ${ }^{\circ}$ journal and benefit from:}

- Convenient online submission

- Rigorous peer review

- Immediate publication on acceptance

- Open access: articles freely available online

- High visibility within the field

- Retaining the copyright to your article 